\title{
Three New Limonoids from Cipadessa cinerascens
}

\author{
Xiao-Hong Yuan, ${ }^{a}$ Bo-Gang Li, ${ }^{b}$ Chun-Xia Xu, ${ }^{a}$ Min Zhou, ${ }^{b}$ Hua-Yi Qı, ${ }^{b}$ and Guo-Lin Zhang ${ }^{*, b}$ \\ ${ }^{a}$ College of Life Science and Engineering, Southwest University of Science and Technology; Mianyang 621010, P. R. \\ China: and ${ }^{b}$ Chengdu Institute of Biology, Chinese Academy of Sciences; Chengdu 610041, P. R. China. \\ Received October 14, 2006; accepted March 3, 2007
}

Three new limonoids, cipadesins $D-F(1-3)$, together with 8,15-dihydroxy-13E-labdane, $\beta$-sitosterol and $\beta$-daucosterol, were isolated from the leaves and bark of Cipadessa cinerascens. Their structures were elucidated by spectral evidence. $X$-Ray crystallographic analysis confirmed the structure of 1 .

Key words Meliaceae; Cipadessa cinerascens; limonoid; cipadesin D; cipadesin E; cipadesin F

The leaves and roots of Cipadessa cinerascens (PELl.) HAND-MAzz (Meliaceae), a shrub distributed in Southwest China, are used for the treatment of rheumatism, malaria, scald and skin itch. ${ }^{1)}$ From the leaves of $C$. cinerascens flavonoids and their glucosides were isolated. ${ }^{2-4)}$ From the leaves and bark of $C$. cinerascens, novel limonoids, cipadesins $\mathrm{A}-\mathrm{C}$, were obtained during our study. ${ }^{5)}$ The continued study led to the isolation of three new limonoids, cipadesins D-F $(\mathbf{1}-\mathbf{3})$, together with 8,15-dihydroxy-13Elabdane, ${ }^{6)} \beta$-sitosterol and $\beta$-daucosterol.

Cipadesin D (1) was isolated as colorless crystal (MeOH). Its molecular formula $\mathrm{C}_{33} \mathrm{H}_{40} \mathrm{O}_{13}$ was established from the quasi-molecular ion peak at $\mathrm{m} / z 667.2330[\mathrm{M}+\mathrm{Na}]^{+}$in the HR-ESI-MS. The IR peaks at 1749 and $1738 \mathrm{~cm}^{-1}$, and ${ }^{13} \mathrm{C}-$ NMR signals at $\delta$ 205.3, 173.7, 170.8, 170.4, 168.8 and 168.1 revealed the presence of a ketonic carbonyl group and five ester carbonyl groups. Besides a methoxy group $\left(\delta_{\mathrm{H}}\right.$ $\left.3.69 ; \delta_{\mathrm{C}} 52.0\right)$ and three acetyl groups $\left(\delta_{\mathrm{H}} 1.87,2.09,2.11\right.$; $\left.\delta_{\mathrm{C}} 168.8,170.4,170.8\right)$, the left twenty six ${ }^{13} \mathrm{C}-\mathrm{NMR}$ signals indicated that 1 may be a limonoid. The presence of $\beta$-substituted furan ring $\left(\delta_{\mathrm{H}} 6.42,7.44,7.59 ; \delta_{\mathrm{C}} 108.8,121.1,140.4\right.$, 143.4) and four tertiary methyl groups as well as the HMBC experiment (Fig. 2) showed that $\mathbf{1}$ was trijugin type limonoid, ${ }^{7-9)}$ characterized by a five-membered ring $C$ with an exocyclic carbonyl at C-9. Three acetyl groups were located at $\mathrm{C}-2, \mathrm{C}-3$ and $\mathrm{C}-12$ by the HMBC cross signals between H-2 $\left(\delta_{\mathrm{H}} 5.25\right)$, OAc $\left(\delta_{\mathrm{C}} 170.4\right)$, between H-3 $\left(\delta_{\mathrm{H}}\right.$ $5.14)$, OAc $\left(\delta_{\mathrm{C}} 170.8\right)$, and between H-12 $\left(\delta_{\mathrm{H}} 5.55\right)$, OAc $\left(\delta_{\mathrm{C}}\right.$ 168.8). The relative stereochemistry of 1 was determined by NOESY experiments (Fig. 2). X-Ray crystallographic analysis of 1 (Fig. 3 ) confirmed the structure.

The UV, IR, ${ }^{1} \mathrm{H}$ - and ${ }^{13} \mathrm{C}-\mathrm{NMR}$ spectral data of cipadesins E, F $(2,3)$ were similar to those of $\mathbf{1}$, suggesting they are all limonoids.

Cipadesin E (2) was isolated as white powder. Its molecular formula $\mathrm{C}_{31} \mathrm{H}_{38} \mathrm{O}_{12}$ was provided by the quasi-molecular

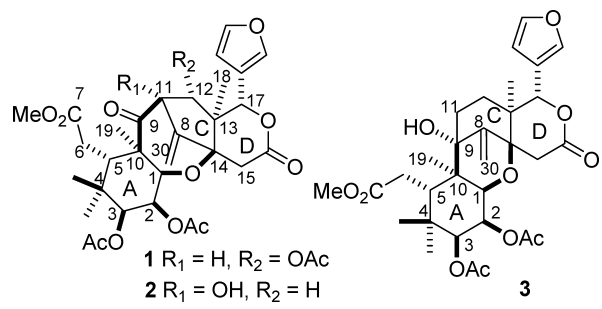

Fig. 1. Cipadesins $\mathrm{D}-\mathrm{F}(\mathbf{1}-\mathbf{3})$ Isolated from Cipadessa cinerascens ion peak at $m / z 625.2283[\mathrm{M}+\mathrm{Na}]^{+}$in the HR-ESI-MS. The presence of a ketonic carbonyl group and four ester carbonyl groups were recognized from the IR peak at $1746 \mathrm{~cm}^{-1}$, and ${ }^{13} \mathrm{C}-\mathrm{NMR}$ signals at $\delta 210.7,173.9,170.8,170.4$ and 168.4. The HMBC correlations of H-12 $(\delta 1.55), \mathrm{H}-19(\delta 1.14)$ and H-30 $(\delta 5.58,5.28)$ with C-9 $(\delta 210.7)$ showed that 2 was trijugin type limonoid (Fig. 2). A hydroxyl group resonated at $\delta$ 4.72 gave HMBC correlations with C-9 and C-11, and NOESY correlation with $\mathrm{H}-12 \alpha$, allowing it to be assigned to $\mathrm{C}-11 \alpha$. The remaining structure was confirmed by HMBC and NOESY experiments (Fig. 2).

Cipadesin F (3) was isolated as white powder. Its molecular formula $\mathrm{C}_{31} \mathrm{H}_{40} \mathrm{O}_{11}$ was concluded from the quasi-molecular ion peak at $\mathrm{m} / \mathrm{z} 611.2471[\mathrm{M}+\mathrm{Na}]^{+}$in the HR-ESI-MS. Four ester carbonyl groups were revealed by the IR peak at $1739 \mathrm{~cm}^{-1}$ and ${ }^{13} \mathrm{C}$-NMR signals at $\delta 175.0,170.7,170.2$ and 169.9. HMBC experiment (Fig. 2) showed that 3 was methyl angolensate type limonoid, ${ }^{10,11)}$ characterized by a six-membered ring $\mathrm{C}$ connected with ring $\mathrm{A}$ by $\mathrm{C}-9$ and $\mathrm{C}$ 10. The structure of $\mathbf{3}$ was determined by NMR data in DMSO- $d_{6}$. The hydroxyl group resonated at $\delta 4.72$ was as-

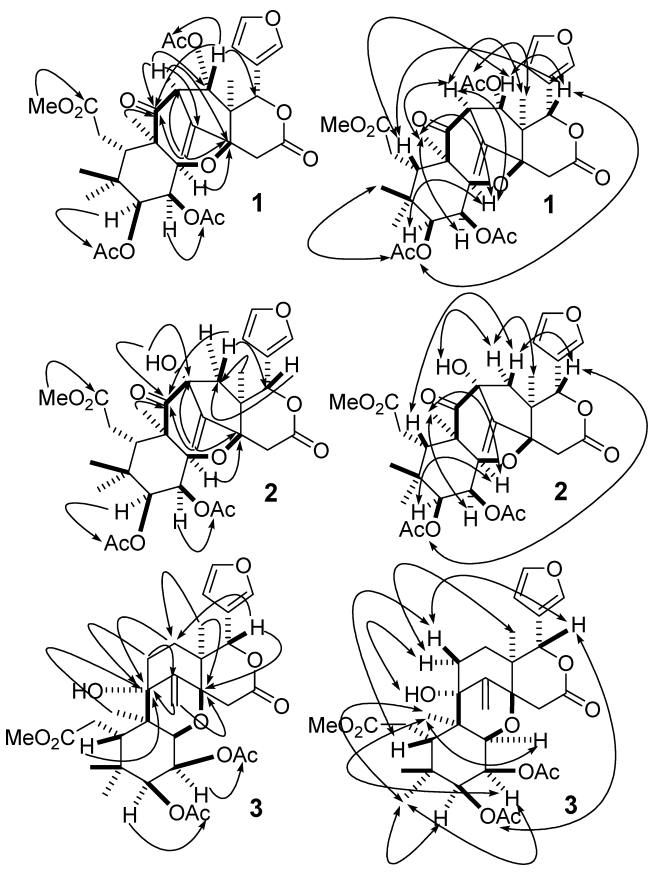

Fig. 2. Important HMBC and NOESY Correlations of $\mathbf{1}-\mathbf{3}$ (HMBC: $\rightarrow$; NOESY: $\leftrightarrow)$ 
Table 1. ${ }^{1} \mathrm{H}-\mathrm{NMR}$ Data of Cipadesins $\mathrm{D}-\mathrm{F}(\mathbf{1}-\mathbf{3})$ in $\mathrm{CDCl}_{3}(600 \mathrm{MHz})$

\begin{tabular}{|c|c|c|c|c|}
\hline Position & 1 (multi., $J$ in $\mathrm{Hz})^{a)}$ & 2 (multi., $J$ in $\mathrm{Hz})^{b}$ ) & 3 (multi., $J$ in $\mathrm{Hz})^{a)}$ & 3 (multi., $J$ in $\mathrm{Hz}$ ) \\
\hline 1 & $4.08(\mathrm{~d}, 3.1)$ & $4.05(\mathrm{~d}, 3.2)$ & $3.48(\mathrm{~d}, 4.6)$ & $3.42(\mathrm{~d}, 4.6)$ \\
\hline 2 & $5.25(\mathrm{dd}, 3.7,3.1)$ & $5.24(\mathrm{dd}, 3.4,3.2)$ & $5.17(\mathrm{dd}, 4.6,3.0)$ & $5.06(\mathrm{dd}, 4.6,3.0)$ \\
\hline 3 & $5.13(\mathrm{~d}, 3.7)$ & $5.14(\mathrm{~d}, 3.4)$ & $5.05(\mathrm{~d}, 3.0)$ & $4.91(\mathrm{~d}, 3.0)$ \\
\hline 5 & $3.13(\mathrm{dd}, 6.9,2.2)$ & $2.95(\mathrm{dd}, 5.9,3.5)$ & 2.91 (overlapped) & $2.79(\mathrm{~d}, 10.0)$ \\
\hline $6 a$ & $2.89(\mathrm{dd}, 18.5,2.2)$ & $2.84(\mathrm{dd}, 18.5,5.9)$ & $3.04(\mathrm{~d}, 17.1)$ & $3.04(\mathrm{~d}, 17.0)$ \\
\hline $6 \mathrm{~b}$ & $2.25(\mathrm{dd}, 18.5,6.9)$ & $2.33(\mathrm{dd}, 18.5,3.5)$ & 2.43 (overlapped) & $2.36(\mathrm{dd}, 17.0,10.0)$ \\
\hline $11 \alpha$ & $3.33(\mathrm{~d}, 2.1)$ & & 1.38 (overlapped) & $1.49(\mathrm{dt}, 14.3,5.8)$ \\
\hline $11 \beta$ & & & $2.73(\mathrm{~m})$ & $2.39(\mathrm{~m})$ \\
\hline $12 \alpha$ & & $1.55(\mathrm{~d}, 14.1)$ & 1.38 (overlapped) & $1.11(\mathrm{~m})$ \\
\hline $12 \beta$ & $5.55(\mathrm{~d}, 2.1)$ & $3.22(\mathrm{~d}, 14.1)$ & 2.38 (overlapped) & 2.23 (overlapped) \\
\hline $15 \alpha$ & $2.85(\mathrm{~s})$ & $2.84(\mathrm{~s})$ & $2.52(\mathrm{~d}, 17.8)$ & $2.24(\mathrm{~d}, 17.8)$ \\
\hline $15 \beta$ & $2.85(\mathrm{~s})$ & $2.84(\mathrm{~s})$ & $2.91(\mathrm{~d}, 17.8)$ & $3.20(\mathrm{~d}, 17.8)$ \\
\hline $17 \beta$ & $6.51(\mathrm{~s})$ & $6.32(\mathrm{~s})$ & $5.79(\mathrm{~s})$ & $5.61(\mathrm{~s})$ \\
\hline 18 & $0.94(\mathrm{~s})$ & $0.98(\mathrm{~s})$ & $0.84(\mathrm{~s})$ & $0.80(\mathrm{~s})$ \\
\hline 19 & $1.12(\mathrm{~s})$ & $1.14(\mathrm{~s})$ & $0.90(\mathrm{~s})$ & $0.76(\mathrm{~s})$ \\
\hline 21 & $7.59(\mathrm{~s})$ & $7.53(\mathrm{~s})$ & $7.43(\mathrm{~s})$ & $7.68(\mathrm{~s})$ \\
\hline 22 & $6.42(\mathrm{~s})$ & $6.42(\mathrm{~d}, 0.8)$ & $6.40(\mathrm{~s})$ & $6.49(\mathrm{~s})$ \\
\hline 23 & $7.44(\mathrm{~s})$ & $7.45(\mathrm{~s})$ & $7.42(\mathrm{~s})$ & $7.71(\mathrm{~s})$ \\
\hline 28 & $1.09(\mathrm{~s})$ & $1.09(\mathrm{~s})$ & $1.12(\mathrm{~s})$ & $1.03(\mathrm{~s})$ \\
\hline 29 & $0.87(\mathrm{~s})$ & $0.90(\mathrm{~s})$ & $0.84(\mathrm{~s})$ & $0.72(\mathrm{~s})$ \\
\hline $30 \mathrm{a}$ & $5.40(\mathrm{~s})$ & $5.58(\mathrm{~s})$ & $5.35(\mathrm{~s})$ & $5.32(\mathrm{~s})$ \\
\hline $30 \mathrm{~b}$ & $5.22(\mathrm{~s})$ & $5.28(\mathrm{~s})$ & $5.01(\mathrm{~s})$ & $5.12(\mathrm{~s})$ \\
\hline $\mathrm{OMe}$ & $3.69(\mathrm{~s})$ & $3.67(\mathrm{~s})$ & $3.70(\mathrm{~s})$ & $3.61(\mathrm{~s})$ \\
\hline $\mathrm{OAc}-2 \beta$ & $2.11(\mathrm{~s})$ & $2.12(\mathrm{~s})$ & 2.00 & 1.85 \\
\hline $\mathrm{OAc}-3 \beta$ & $2.09(\mathrm{~s})$ & $2.08(\mathrm{~s})$ & 2.15 & 2.12 \\
\hline OAc- $12 \alpha$ & $1.87(\mathrm{~s})$ & & & \\
\hline
\end{tabular}

a) Assignments were based on HSQC and HMBC experiments, $b$ ) assignments were based on HMQC and HMBC experiments, $c$ ) in DMSO- $d_{6}$.

Table 2. ${ }^{13} \mathrm{C}-\mathrm{NMR}$ Data of Cipadesins $\mathrm{D}-\mathrm{F}(\mathbf{1}-\mathbf{3})$ in $\mathrm{CDCl}_{3}(150 \mathrm{MHz})$

\begin{tabular}{|c|c|c|c|c|}
\hline C-atom & $\mathbf{1}^{a)}$ & $\mathbf{2}^{b)}$ & $3^{a)}$ & $\mathbf{3}^{a, c)}$ \\
\hline 1 & 74.0 & 73.6 & 74.8 & 74.2 \\
\hline 2 & 65.5 & 65.3 & 66.4 & 67.1 \\
\hline 3 & 74.5 & 74.2 & 76.2 & 76.2 \\
\hline 4 & 39.2 & 39.1 & 39.2 & 39.2 \\
\hline 5 & 35.6 & 37.2 & 36.3 & 36.2 \\
\hline 6 & 28.4 & 29.3 & 31.1 & 31.0 \\
\hline 7 & 173.7 & 173.9 & 175.0 & 174.8 \\
\hline 8 & 142.2 & 147.0 & 149.2 & 149.4 \\
\hline 9 & 205.3 & 210.7 & 78.6 & 77.7 \\
\hline 10 & 56.5 & 53.4 & 50.7 & 50.3 \\
\hline 11 & 69.1 & 85.9 & 34.8 & 33.3 \\
\hline 12 & 76.5 & 47.2 & 30.2 & 30.6 \\
\hline 13 & 49.5 & 45.0 & 41.2 & 41.0 \\
\hline 14 & 88.2 & 87.3 & 81.7 & 81.8 \\
\hline 15 & 33.8 & 33.7 & 33.7 & 34.3 \\
\hline 16 & 168.1 & 168.4 & 169.9 & 170.1 \\
\hline 17 & 78.3 & 79.5 & 80.0 & 79.9 \\
\hline 18 & 11.5 & 17.6 & 13.6 & 13.9 \\
\hline 19 & 19.7 & 19.3 & 16.2 & 16.8 \\
\hline 20 & 121.1 & 121.8 & 121.2 & 121.7 \\
\hline 21 & 140.4 & 139.7 & 140.1 & 140.1 \\
\hline 22 & 108.8 & 108.5 & 109.5 & 110.4 \\
\hline 23 & 143.4 & 143.6 & 143.0 & 144.0 \\
\hline 28 & 22.8 & 22.8 & 21.7 & 21.7 \\
\hline 29 & 27.4 & 27.6 & 27.1 & 27.4 \\
\hline 30 & 114.8 & 114.1 & 109.5 & 110.4 \\
\hline $\mathrm{OCH}_{3}$ & 52.0 & 52.0 & 51.9 & 52.0 \\
\hline OAc-2 & $170.4,20.8$ & $170.4,20.8$ & $170.2,21.4$ & $170.0,20.9$ \\
\hline OAc-3 & $170.8,20.4$ & $170.8,20.4$ & $170.7,20.8$ & $170.3,21.4$ \\
\hline OAc-12 & $168.8,20.4$ & & & \\
\hline
\end{tabular}

a) Assignments were based on HSQC and HMBC experiments, $b$ ) assignments were based on HMQC and HMBC experiments, $c$ ) in DMSO- $d_{6}$.

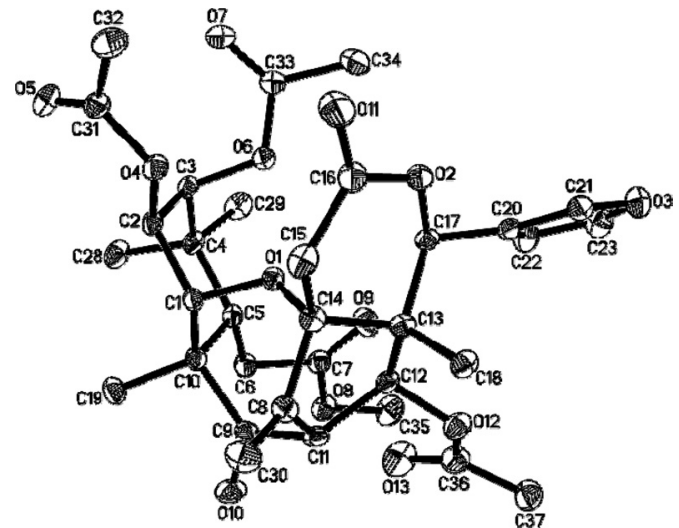

Fig. 3. ORTEP Diagram of 1

signed as HO- $9 \alpha$ on the basis of its correlations with C-8, C9, $\mathrm{C}-10$ and $\mathrm{C}-11$ in $\mathrm{HMBC}$ experiment, and with $\mathrm{H}-11 \alpha$ in NOESY experiment (Fig. 2). Two acetyl groups were placed at $\mathrm{C}-2$ and $\mathrm{C}-3$ by the HMBC cross signals between $\mathrm{H}-2$, $\mathrm{OAc}$, and between $\mathrm{H}-3, \mathrm{OAc}$. The relative stereochemistry of 3 was determined by NOESY experiment.

\section{Experimental}

General Melting points were measured on an X-6 melting point apparatus and are uncorrected. Optical rotations were measured on a Perkin-Elmer 341 automatic polarimeter. UV and IR spectra were recorded on a Lambda 35 spectrometer and a Perkin-Elmer FT-IR spectrometer ( $\mathrm{KBr}$ disk), respectively. Mass spectra were obtained on a Finnigan-LCQ ${ }^{\mathrm{DECA}}$ mass spectrometer (ESI-MS) and a Bruker Daltonics Bio TOF-Q mass spectrometer (HRESI-MS). NMR spectra were carried out on a Bruker Avance 600 spectrometer with TMS as internal standard. Column chromatography was carried out on silica gel (200-300 mesh; Qingdao Haiyang Chemical Group Co., China), silica gel $60(0.015-0.040 \mathrm{~mm}$, Merck), MCI gel $(75-150 \mu \mathrm{m}$, Mitsubishi) and RP-18 silica gel (Prepex 40-63 $\mu \mathrm{m}$, Phenomenex). Semi- 
preparative HPLC was carried out using a Perkin Elmer Series 200 HPLC system (Lichrocart RP-C ${ }_{18}$ column, $10 \times 250 \mathrm{~mm}, 5 \mu \mathrm{m}$ ). Solvents were distilled prior to use.

Plant Material Leaves and bark of Cipadessa cinerascens were collected in May 2004 at Panzhihua City, Sichuan Province of China, and identified by Prof. Zuo-Cheng Zhao at Chengdu Institute of Biology of the Chinese Academy of Sciences (CAS). A voucher specimen (No. A-186) was deposited in the Natural Products Research Center at Chengdu Institute of Biology, CAS.

Extraction and Isolation Air-dried and powdered leaves and bark $(4.8 \mathrm{~kg})$ were percolated with $95 \% \mathrm{EtOH}(401 \times 2$, each $7 \mathrm{~d})$ at room temperature. After evaporation of the solvents in vacuo at $50^{\circ} \mathrm{C}$, a residue $(310 \mathrm{~g})$ was obtained. This residue was suspended in $\mathrm{H}_{2} \mathrm{O}$ (11) and extracted with $\mathrm{CHCl}_{3}(11 \times 3)$. The $\mathrm{CHCl}_{3}$ extract $(75 \mathrm{~g})$ was partitioned between $95 \%$ aqueous $\mathrm{MeOH}$ and petroleum ether $\left(60-90^{\circ} \mathrm{C}\right)$ to yield the $\mathrm{MeOH}$ soluble fraction $(45 \mathrm{~g})$. $\beta$-Daucosterol ( $4 \mathrm{~g})$ was obtained by crystallization of $\mathrm{MeOH}$ soluble fraction from $\mathrm{MeOH}$, and the remnant was then subjected to silica gel column $(\phi 8 \times L 25 \mathrm{~cm}, 800 \mathrm{~g})$ and eluted with $\mathrm{CHCl}_{3}-\mathrm{EtOAc}$ $\mathrm{MeOH}(25: 5: 1)$ to give fractions $\mathrm{A}-\mathrm{C}(\mathrm{A}, 1.3 \mathrm{~g} ; \mathrm{B}, 12.2 \mathrm{~g} ; \mathrm{C}, 2.5 \mathrm{~g}) . \beta$ Sitosterol $(580 \mathrm{mg})$ was obtained by crystallization of fraction A from $\mathrm{MeOH}$. Fraction B was subjected to MCI gel column $(\phi 5 \times L 12 \mathrm{~cm}, 150 \mathrm{~g})$ and eluted with a gradient mixture of $\mathrm{H}_{2} \mathrm{O}$ and $\mathrm{MeOH}(1: 1,3: 7,1: 9,0: 1$, each 21$)$ to give fractions BA-BD. $1(310 \mathrm{mg})$ was obtained by crystallization of fraction $\mathrm{BB}(2.7 \mathrm{~g})$ from $\mathrm{MeOH}$, and the remnant was then subjected to silica gel column $(\phi 5 \times L 20 \mathrm{~cm}, 300 \mathrm{~g})$ and eluted with $\mathrm{CHCl}_{3}-\mathrm{EtOAc}-$ $\mathrm{MeOH}(50: 5: 1)$ to give fractions BBA-BBD. Fraction BBA $(1.50 \mathrm{~g})$ was further separated over silica gel 60 column $(\phi 3.5 \times L 15 \mathrm{~cm}, 80 \mathrm{~g})$ using petroleum ether $\left(60-90^{\circ} \mathrm{C}\right)$-acetone $(4: 1)$ to give fractions BBAA and BBAB. Fraction BBAA was subjected to RP-18 silica gel column $(\phi 2 \times L$ $20 \mathrm{~cm}, 40 \mathrm{~g})$ and eluted with water-methanol $(2: 3)$ to give white powder $(160 \mathrm{mg})$, which was divided into subfractions $1-3$ by reversed phase HPLC (56\% aqueous MeOH, $3 \mathrm{ml} / \mathrm{min}, 227 \mathrm{~nm}) .2(9 \mathrm{mg})$ and $3(12 \mathrm{mg})$ were obtained from subfraction $1(28 \mathrm{mg})$ by $\mathrm{HPLC}\left(47 \%\right.$ aqueous $\mathrm{CH}_{3} \mathrm{CN}$, $3 \mathrm{ml} / \mathrm{min}, 227 \mathrm{~nm})$. Fraction BBB (170 mg) was further separated over silica gel 60 column $(\phi 2 \times L 17 \mathrm{~cm}, 30 \mathrm{~g})$ using petroleum ether $\left(60-90^{\circ} \mathrm{C}\right)$-acetone $(6: 1)$ to give yellow oily residue $(50 \mathrm{mg})$, from which 8,15 -dihydroxy$13 E$-labdane $(20 \mathrm{mg})$ was obtained by crystallization from $\mathrm{MeOH}$ at $4{ }^{\circ} \mathrm{C}$.

Cipadesin D (1): Colorless needles (MeOH). mp 208.1-209.0 ${ }^{\circ} \mathrm{C}$. ${ }^{1} \mathrm{H}$ NMR data see Table $1 .{ }^{13} \mathrm{C}-\mathrm{NMR}$ data see Table 2. IR $(\mathrm{KBr}) \mathrm{cm}^{-1}: 3445$, 2990, 1749, 1738, 1686, 1372, 1244, 1073, 1003, 875, 607. UV $\lambda_{\text {max }}$ $\left(\mathrm{CHCl}_{3}\right) \mathrm{nm}(\log \varepsilon): 241$ (2.87). HR-ESI-MS (positive mode) $\mathrm{m} / z 667.2330$ (Calcd for $\left.\mathrm{C}_{33} \mathrm{H}_{40} \mathrm{O}_{13} \mathrm{Na}[\mathrm{M}+\mathrm{Na}]^{+}, 667.2361\right)$. $[\alpha]_{\mathrm{D}}^{25}-5.3^{\circ},[\alpha]_{578}^{25}-5.3^{\circ}$, $[\alpha]_{546}^{25}-7.2^{\circ},[\alpha]_{436}^{25}-28.9^{\circ},[\alpha]_{365}^{25}-157.9^{\circ}\left(c=0.15, \mathrm{CHCl}_{3}\right)$.

Cipadesin E (2): White powder. ${ }^{1} \mathrm{H}-\mathrm{NMR}$ data see Table $1 .{ }^{13} \mathrm{C}-\mathrm{NMR}$ data see Table 2. IR (KBr) $\mathrm{cm}^{-1}: 3445,2976,1746,1686,1634,1379,1248$, 1164, 1083, 1048, 1021, 876, 602. UV $\lambda_{\max }\left(\mathrm{CHCl}_{3}\right) \mathrm{nm}(\log \varepsilon): 240$ (2.03). HR-ESI-MS (positive mode) $m / z \quad 625.2283$ (Calcd for $\mathrm{C}_{31} \mathrm{H}_{38} \mathrm{O}_{12} \mathrm{Na}$ $\left.[\mathrm{M}+\mathrm{Na}]^{+}, 625.2255\right) .[\alpha]_{\mathrm{D}}^{25}-6.1^{\circ},[\alpha]_{578}^{25}-6.4^{\circ},[\alpha]_{546}^{25}-7.4^{\circ},[\alpha]_{436}^{25}-10.2^{\circ}$, $[\alpha]_{365}^{25}-16.8^{\circ}\left(c=0.39, \mathrm{CHCl}_{3}\right)$.

Cipadesin F (3): White powder. ${ }^{1} \mathrm{H}-\mathrm{NMR}$ data see Table $1 .{ }^{13} \mathrm{C}-\mathrm{NMR}$ data see Table 2. IR (KBr) $\mathrm{cm}^{-1}: 3451,2953,1739,1635,1384,1250,1198$, 1168, 1049, 875, 602. UV $\lambda_{\max }\left(\mathrm{CHCl}_{3}\right) \mathrm{nm}(\log \varepsilon): 240$ (1.89). ESI-MS (positive mode) $\mathrm{m} / \mathrm{z} 611[\mathrm{M}+\mathrm{Na}]^{+}(100)$. HR-ESI-MS (positive mode) $\mathrm{m} / \mathrm{z}$ 611.2471 (Calcd for $\left.\mathrm{C}_{31} \mathrm{H}_{40} \mathrm{O}_{11} \mathrm{Na}[\mathrm{M}+\mathrm{Na}]^{+}, 611.2463\right) .[\alpha]_{\mathrm{D}}^{25}-36.5^{\circ},[\alpha]_{578}^{25}$ $-38.2^{\circ},[\alpha]_{546}^{25}-43.5^{\circ},[\alpha]_{436}^{25}-74.8^{\circ},[\alpha]_{365}^{25}-118.8^{\circ}\left(c=0.42, \mathrm{CHCl}_{3}\right)$.

X-Ray Crystallography of 1 Crystal data: $\mathrm{C}_{33} \mathrm{H}_{40} \mathrm{O}_{13} ; M_{\mathrm{W}}=644.65$; dimensions $0.48 \times 0.48 \times 0.46 \mathrm{~mm}$; monoclinic system, space group $P 2_{1} 2_{1} 2_{1}$, $a=10.770$ (4) $\AA, b=11.174(3) \AA, c=13.377$ (4) $\AA, \alpha=\beta=\gamma=90^{\circ}, V=1609.6$ (11) $\AA^{3}, Z=2, d=1.330 \mathrm{~g} / \mathrm{cm}^{-3}, \lambda=0.71073 \AA, \mu(\mathrm{Mo} \mathrm{KR})=0.089 \mathrm{~mm}^{-1}, F$ $(000)=684, T=296(2) \mathrm{K}$. Of the 4231 reflections collected, 3886 were unique $\left(R_{\text {int }}=0.0064\right)$. The structure was solved by direct method with SHELX $97^{12)}$ and refined by fullmatrix least-squares on $F^{2}$. Final refinement: data/restraints/parameters $=3886 / 1 / 424 ; R 1=0.0644$ (all data), $w R 2=$ 0.1084 (all data); the Flack absolute structure parameter $=0$ (10), and $\mathrm{GOF}=0.945$. The $\mathrm{H}$ coordinates were determined by calculated geometry. The maximum and minimum peaks on the final difference Fourier map corresponded to 0.188 and $-0.228 \mathrm{e}^{-} / \AA^{3}$, respectively. CCDC 278633 contains the supplementary crystallographic data for this paper. These data can be obtained free of charge via www.ccdc.cam.ac.uk, or by contacting The Cambridge Crystallographic Data Centre, 12 Union Road, Cambridge, CB2 1EZ, U.K., fax: +44 1223336033 .

Supporting Information Available X-Ray data of 1; HR-ESI-MS spectra; $1 \mathrm{D}$ and 2D NMR diagrams of $\mathbf{1}-\mathbf{3}$. This material is available free of charge via the Internet at http://cpb.pharm.or.jp.

Acknowledgements This work was financial supported by Di-Ao Pharmaceutical Group in Chengdu. The authors are grateful to $\mathrm{Mr}$. Jian $\mathrm{Gu}$ at Chengdu Institute of Biology of the Chinese Academy of Sciences for collecting plant material.

\section{References}

1) "A Dictionary of Chinese Herb," ed. by Jiangsu New Medical College, Shanghai People Press, Jiangsu, 1977, p. 2183.

2) Liang L., Zhong C. C., Xiao Z. Y., Zhongcaoyao, 21, $2-4$ (1990).

3) Liang L., Zhong C. C., Xiao Z. Y., Zhongcaoyao, 22, 6-8 (1991).

4) Liang L., Zhong C. C., Xiao Z. Y., Zhongcaoyao, 25, 236-237 (1994).

5) Yuan X. H., Li B. G., Zhou M., Qi H. Y., Zhang G. L., Org. Lett., 7, 5051-5053 (2005).

6) Rojatkar S. R., Chiplunkar Y. G., Nagasampagi B. A., Phytochemistry, 37, 1213-1214 (1994).

7) Mulholland D. A., Lourine S. E., Phytochemistry, 47, 1357-1361 (1998).

8) Kehrli A. R. H., Taylor D. A. H., Miven M., Phytochemistry, 29, 153-159 (1990).

9) Purushothaman K. K., Venkatanarasimhan M., Sarada A. L., Connolly J. D., Rycroft D. D., Can. J. Chem., 65, 35 (1987).

10) Chan W. R., Magnus K. E., Mootoo B. S., J. Chem. Soc. C, 1967, 171-177 (1967).

11) Taylor D. H. A., J. Chem. Soc., Chem. Commun., 1969, 58 (1969).

12) Sheldrick G. M., "SHELXS-97: Manual of Program for the Refinement of Crystal Structures," University of Göttingen, Göttingen, Germany, 1997. 